ORIGINAL ARTICLE

\title{
Sonographic Association of Ventriculomegaly and Neural Tube Anomalies
}

\author{
SHAMSHAD YOUSAF ${ }^{1}$, SYEDA KHADIJA ${ }^{1}$, NAVEED ASAD ${ }^{1}$, MEHREEN FATIMA ${ }^{1}$, SYED AMIR GILANI ${ }^{1}$, TALHA \\ LAIQUE $^{2}$ \\ ${ }^{1}$ Department of Radiological Sciences and Medical Imaging Technology, University of Lahore, Lahore-Pakistan \\ ${ }^{2}$ Department of Pharmacology, Lahore Medical and Dental College, Lahore-Pakistan \\ Correspondence to Dr. Talha Laique, Email: talhalaique51@gmail.com Tel:+92-331-0346682
}

\begin{abstract}
Background: Ventriculomegaly is most common indicator of many of neural tube anomalies of intrauterine fetus. Presence of which has alarming sign for sonologist to have careful evaluation of fetus for other cranial and extra cranial anomalies.

Aim: To evaluate the sonographic difference of fetal ventricle dilatation and neural tube anomalies.

Study design: Cross Sectional study.

Methodology: Female patients $(n=1492)$ were enrolled in present study held at University Ultrasound Clinic, Green Town, University of Lahore as a reference placement for 6 months. Informed consent was taken from all of them. In this study, pregnant women who underwent ultrasound scanning between 18 and 40 weeks of gestational age were reviewed according to normal size of ventricles and dilatation of ventricles by per abdominal ultrasound having convex transducer with $3.5 \mathrm{MHZ}$ frequency.

Results: A total of 1492 cases of pregnant women between gestational ages of 18-40 weeks were examined. Normal cases were 1485/1492(99.5\%).Total 7-cases were found to have ventricle dilatation. Fetus with mild ventricular dilatation 1/7, with moderate ventricle dilatation were 4/7, 2/4 having moderate ventricle dilatation were associated with neural tube anomalies, severe ventricle dilatation was noted in 2/7, both these fetuses had neural tube anomalies.

Conclusion: This study concluded that level of degree of dilatation increases the chances of abnormalities. Early detection provide better counseling opportunity for family to make decisions regarding their fetuses.

Keywords: Neural tube defects, Ventriculomegaly, Gestation age and Ultrasonography.
\end{abstract}

\section{INTRODUCTION}

Ventriculomegaly is defined as dilatation of the lateral ventricles of the growing intrauterine baby however $10 \mathrm{~mm}$ size of lateral ventricle is taken as normal. It is confirmed when the size of one or bilateral ventricle measurement is more than $10 \mathrm{~mm} .^{1}$ Dilatation of the ventricle incidence is 1-2 / 1000 who are born alive. ${ }^{2}$ Categories of ventricle dilatation can be done by two ways: mild $10-15 \mathrm{~mm}$ or severe more than $15 \mathrm{~mm}$; or as mild (10-12 $\mathrm{mm})$, moderate $(13-15 \mathrm{~mm}) .^{3}$ Severe category of ventricle dilatation is measurement of $15-17 \mathrm{~mm}$ in the trans ventricular plane. ${ }^{4}$ Cerebral ventricles appear in brain from 15 weeks, and normal lateral ventricle retain the same size of $10 \mathrm{~mm}$ throughout their gestational period. ${ }^{5}$ ventricle dilatation can occur from a number abnormal factors which consists of blockage of normal flowing pattern of cerebrospinal fluid formed by choroid plexus of lateral ventricles, decreased size of brain parenchyma due to fetal infection, bleeding in brain, Genetically transmitted brain anomalies, or by structural defects of posterior fossa ${ }^{6,7}$. Collective term for Spinal cord defects, absence of normal brain tissues, and presence of cystic lesion at nape of neck are neural tube defects ${ }^{8}$. An evaluation of the intra uterine baby consists of ultrasound examination of the ventricles of brain, and their measurement of size of ventricles is major part of the central nervous system evaluation ${ }^{9}$. The finding of fetal ventricle dilatation are different in multiple cases especially when are in association with other fetal

Received on 23-12-2020
Accepted on 27-04-2021

anomalies ${ }^{10}$. Hydrocephalus is also dilatation of ventricles but above ventricle dilatation of $17 \mathrm{~mm}$ along with increase in the head circumference size ${ }^{11}$. In adults Long-standing overt ventricle dilatation is special type of ventricle dilatation that grow in childhood and show its features during adult life. ${ }^{12}$ Although the confirmation of ventricle dilatation is easy process at ultrasound by measuring size of lateral ventricles but to rule out cause of ventricle dilatation is much difficult in intra uterine period ${ }^{13}$. Chances the of smaller and greater abnormalities increases with each millimeter increasing in size of ventricle ${ }^{14}$. Better prognoses of normal mental growth is noted in babies with mild, static isolated ventricle dilatation or cases in which ventricle dilatation has reduced and returned to its normal size in intrauterine life. ${ }^{15}$ Isolated Ventricle dilatation was associated with better outcome than complex group of ventricle dilatation. ${ }^{16}$ Mild ventricle dilatation detected by ultrasound has good outcome as compared to than the severe degree of the ventricle dilatation as these are associated with other malformations ${ }^{17}$. Severe ventricle dilatation has a worse outcome before birth and neurological and structural and functional disabilities after birth life in majority of babies ${ }^{18}$. Three diagnostic points are mostly related with bad prognoses of baby even in absence of associated abnormalities having ventricle size greater than $12 \mathrm{~mm}$, continuous increase in dilatation of ventricles, asymmetric and dilatation in both ventricles ${ }^{19}$ When ventricle dilatation is detected serial ultrasound of fetus should be done to note any further enlargement in size and any associated anomalies, mother serology. ${ }^{20,21}$ 
Karyotyping is mandatory in the mid trimester of intrauterine baby with moderate lateral ventricle dilatation even there is no other abnormality, Mother serum screening help to detect infections. ${ }^{22}$ During antenatal period evaluation of ventricle dilatation helps in before and just after birth management by providing chance of counseling to parents, so that they can make a decision, about pregnancy continuation or termination, time of termination, method of termination, and site of termination to optimize outcome 23 .

The objective of the study was to evaluate the sonographic difference of fetal ventricle dilatation and neural tube anomalies.

\section{METHODOLOGY}

Female patients $(n=1492)$ were enrolled in present study held at University Ultrasound Clinic,Green Town, University of Lahore as a reference placement for 6 months. Informed consent was taken from all of them. In this study, pregnant women who underwent ultrasound scanning between 18 and 40 weeks of gestational age were reviewed according to normal size of ventricles and dilatation of ventricles by per abdominal ultrasound having convex transducer with 3.5MHZ frequency. Pregnant women were divided in to three groups depending upon the size of ventricle dilatation. A complete and rigorous ultrasound examination was done to search for associated anomalies of the central nervous system and extra cerebral anomalies. Measurement of The plane selected was the transventricular plane. The calipers were placed on the inner borders of the near and far side of the ventricle just within the echogenic lines. The measurement was made on an axis perpendicular to the long axis of the lateral ventricle at the level of the glomus of the choroid plexus. BPD was taken. Measurement of all dilated ventricles and that of normal ventricles were taken.

Statistical analysis: Data analyzed by SPSS 22.0v. Mean \pm SD was used for severity of disease and ventricular dilatation. Frequency and percentage were given for neural tube defects.

\section{RESULTS}

Total of 1492 pregnant women participated in present study, $7(0.5 \%)$ of them have abnormal ventricle dilatation while $1485(99.5 \%)$ have normal size of ventricles as summarized in Table-1. Total of 1492 pregnant women participated in present study, $4(0.2 \%)$ of them have neural tube defects as summarized in Table-2. Total of 1492 pregnant women participated in present study, $7(0.5 \%)$ of them have mild to severe ventricular dilatations as summarized in Table-3. Among all enrolled patients, measurement of ventricular dilatation was presented as Mean \pm SD in table-4.

Table-1: Distribution of normal \& abnormal cases among enrolled subjects

\begin{tabular}{|l|c|c|}
\hline & Frequency & \%age \\
\hline Abnormal & 7 & 0.5 \\
\hline Normal & 1485 & 99.5 \\
\hline Total & 1492 & 100.0 \\
\hline
\end{tabular}

Table-2: Distribution Of Neural Tube Defects Among Enrolled Subjects

\begin{tabular}{|l|c|c|}
\hline & Frequency & \%age \\
\hline Meningocele & 2 & 0.1 \\
\hline Normal & 1488 & 99.8 \\
\hline spina bifida & 2 & 0.1 \\
\hline Total & 1492 & 100.0 \\
\hline
\end{tabular}

Table-3: Distribution of neural tube defects among enrolled subjects

\begin{tabular}{|l|c|c|}
\hline & Frequency & \%age \\
\hline Mild ventricle dilatation & 1 & 0.1 \\
\hline Moderate ventricle dilatation & 4 & 0.3 \\
\hline Severe ventricle dilatation & 2 & 0.1 \\
\hline Total & 1492 & 100.0 \\
\hline
\end{tabular}

Table 4: Measurement Of Ventricular Dilatation $(n=1492)$

\begin{tabular}{|l|c|}
\hline Variables & Mean \pm SD \\
\hline Mild & $10.7000 \pm 0.12$ \\
\hline Moderate & $12.6250 \pm 0.98$ \\
\hline Normal & $9.4342 \pm 2.49$ \\
\hline Severe & $16.1000 \pm 0.14$ \\
\hline Total & $9.4525 \pm 2.50$ \\
\hline
\end{tabular}

Figure-1: Depicting mean of normal, mild, moderate, and severe ventricle dilatations on Sonography

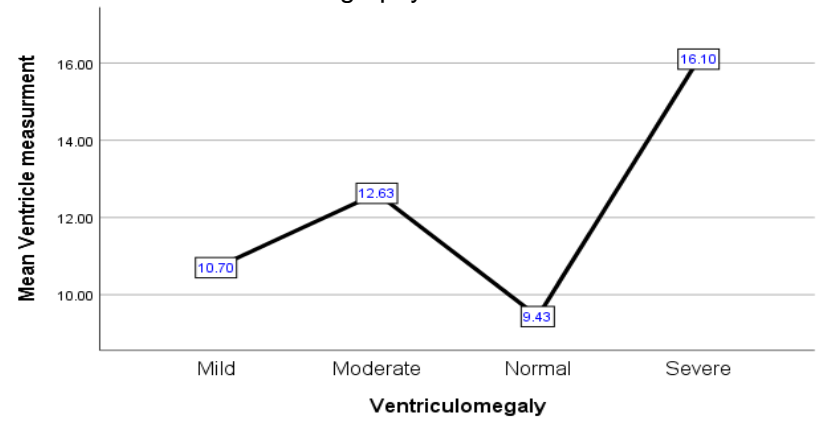

\section{DISCUSSION}

It was a cross sectional study objective of research work was to study the sonographic association of ventricular dilatation and neural tube anomalies. Ventriculomegaly is enlargement in normal diameter of one or both lateral ventricles. $10 \mathrm{~mm}$ is measured normal ventricle size which can be detected at ultrasound from 18 weeks of gestation and size of it remain stable throughout life. It is one of major sign of most of neural tube anomalies. According to study by Coleman BG et al in 2015, with the topic advent of high quality machines with good resolution capacity most of anomalies of fetus can be easily recognized especially structural, cranial and extra cranial. Ultrasound can easily recognize the bony and soft tissue differentiation it can identify any anomalies of vertebras and spinal cords. ${ }^{24}$ In my study, ventricle dilatation and related anomalies mostly detected and diagnosed at by ultrasound evaluation, cases of meningocele and spina bifida associated with ventriculomegaly detected only by ultrasound examination. So ultrasound is best tool for detection of most of 
anomalies. In case of detection of ventriculomegaly in intrauterine life complete work up of fetus must be carried out to rule out its cause and specially when there are associated anomalies, Study by Chen $Q$ et al describes that screening of blood serum of mother can evaluate chances of chromosomal anomalies of the fetus at 21 weeks of intrauterine life as well as presence of any congenital infection ${ }^{25}$. Babies having mild ventricle dilatation ranging from $10-12 \mathrm{~mm}$ mostly have good prognoses when not associated with other neural tube anomalies, ventricle dilatation returns to normal size and does not show any increase of size of enlargement as in my study mild ventricle dilatation is not associated with any associated anomal, same finding were in study by Bromley $B$, and others who recruited forty four intra uterine babies, 17 of these fetuses had other neural tube anomalies. Conclusion was that in those fetuses who have mild ventricular dilatation have fewer chances of related anomalies of fetuses and good prognosis after delivery as compared to severe ventricular dilatation ${ }^{26}$. The chances of related anomalies are greater in severe ventricle dilatation group in which range of dilatation between $15-17 \mathrm{~mm}$ and less in mild ventricle dilatation group as in my study moderate and severe ventricle dilatation of ventricles have more association with neural anomalies and have late neurodevelopment. Similar conclusion was seen in 2014 by Pagani $G$ et al in retrospective study, twenty cases were included in systemic review that assessed pravelance of late neurodevelopment was in $7.9 \%$ cases in which isolated ventricular dilatation was identified. ${ }^{27}$ Results of my study showed the same finding as most of previously published articles and studies regarding association of ventricle dilatation degree to neural tube anomalies.

\section{CONCLUSION}

This study concluded that chances of neural tube abnormalities are higher with each millimeter increasing the diameter of ventricle measurement. As no case of neural tube anomaly was reported with normal ventricle measurement. Mostly babies having normal ventricle and with mild ventricle dilatation ranging from $10-12 \mathrm{~mm}$ mostly have good prognosis when not associated with other neural tube anomalies, ventricle dilatation returns to normal size and does not show any increase of size of enlargement. Early detection provide better counseling opportunity for family to make decisions regarding their fetuses.

Author's contribution: SY \& SK: Overall supervision, write up and literature review. NA \& MF: Statistics application analysis literature review, help in write up. SAG \& TL: Literature review help in write-up.

Acknowledgements: I am thankful to Allah and my colleagues who made it possible for me.

Conflict of interest: None

Funding: None

\section{REFERENCES}

1. D'addario V, Rossi A, editors. Neuroimaging of ventriculomegaly in the fetal period. Seminars in Fetal and Neonatal Medicine; 2012: Elsevier

2. McKechnie L, Vasudevan C, Levene M, editors. Neonatal outcome of congenital ventriculomegaly. Seminars in Fetal and Neonatal Medicine; 2012: Elsevier.

3. Mari G, Norton ME, Stone J, Berghella V, Sciscione A, Tate D, et al.
Seven recommendations for mild fetal ventriculomegaly. Contemporary OB/GYN. 2018:63(8):9-32.

4. Katz JA, Chock VY, Davis AS, Blumenfeld YJ, Hahn JS, Barnes P, et al. Utility of prenatal MRI in the evaluation and management of fetal ventriculomegaly. Journal of Perinatology. 2018;38(11):1444-52.

5. Wax JR, Bookman L, Cartin A, Pinette MG, Blackstone J. Mild fetal cerebral ventriculomegaly: diagnosis, clinical associations, and outcomes. Obstetrical \& gynecological survey. 2003;58(6):407-14.

6. Coady AM, Bower S. Twining's Textbook of Fetal Abnormalities E-Book: Elsevier Health Sciences; 2014

7. Mehlhorn AJ, Morin CE, Wong-You-Cheong JJ, Contag SA. Mild fetal cerebral ventriculomegaly: prevalence, characteristics, and utility of ancillary testing in cases presenting to a tertiary referral center. Prenatal Diagnosis. 2017;37(7):647-57.

8. Padmanabhan R. Etiology, pathogenesis and prevention of neural tube defects. Congenital anomalies. 2006;46(2):55-67.

9. Gaglioti P, Danelon D, Bontempo S, Mombro M, Cardaropoli S, Todros T. Fetal cerebral ventriculomegaly: outcome in 176 cases. Ultrasound in Obstetrics and Gynecology: The Official Journal of the International Society of Ultrasound in Obstetrics and Gynecology. 2005;25(4):372-7.

10. Pagani G, Thilaganathan B, Prefumo F. Neurodevelopmental outcome in isolated mild fetal ventriculomegaly: systematic review and meta-analysis. Ultrasound in Obstetrics \& Gynecology. 2014;44(3):25460.

11. Bannister C, Russell SA, Rimmer S, Arora A. Pre-natal ventriculomegaly and hydrocephalus. Neurological research. 2000;22(1):37-42.

12. Oi S, Shimoda M, Shibata M, Honda $Y$, Togo K, Shinoda M, et al. Pathophysiology of long-standing overt ventriculomegaly in adults. Journal of neurosurgery. 2000;92(6):933-40.

13. D'Addario V, Pinto V, Di Cagno L, Pintucci A. Sonographic diagnosis of fetal cerebral ventriculomegaly: an update. The Journal of MaternalFetal \& Neonatal Medicine. 2007;20(1):7-14.

14. Barzilay E, Bar-Yosef O, Dorembus S, Achiron R, Katorza E. Fetal brain anomalies associated with ventriculomegaly or asymmetry: an MRIbased study. American Journal of Neuroradiology. 2017;38(2):371-5.

15. Gupta JK, Bryce FC, Lilford RJ. Management of apparently isolated fetal ventriculomegaly. Obstetrical \& gynecological survey. 1994;49(10):716-21.

16. Chang $Q$, Peng $Y$, Huang $Q$, Zhong M, Huang L, Li J, et al. Prognosis of fetuses with ventriculomegaly: An observational retrospective study. Prenatal Diagnosis. 2019;39(10):901-9.

17. Joó JG, Tóth Z, Beke A, Papp C, Tóth-Pál E, Csaba Á, et al. Etiology, prenatal diagnostics and outcome of ventriculomegaly in 230 cases. Fetal diagnosis and therapy. 2008;24(3):254-63.

18. Kennelly M, Cooley S, McParland P. Natural history of apparently isolated severe fetal ventriculomegaly: perinatal survival and neurodevelopmental outcome. Prenatal Diagnosis: Published in Affiliation With the International Society for Prenatal Diagnosis. 2009;29(12):1135-40.

19. Ouahba J, Luton D, Vuillard E, Garel C, Gressens P, Blanc N, et al. Prenatal isolated mild ventriculomegaly: outcome in 167 cases. BJOG: An International Journal of Obstetrics \& Gynaecology. 2006;113(9):1072-9.

20. Girard N, Ozanne A, Chaumoitre K, Sigaudy S, Gire C, Dubuc M, et al. $\mathrm{MRI}$ and in utero ventriculomegaly. Journal de radiologie. 2003;84(12 Pt 1):1933.

21. Fox NS, Monteagudo A, Kuller JA, Craigo S, Norton ME, Medicine SfMF. Mild fetal ventriculomegaly: diagnosis, evaluation, and management. American journal of obstetrics and gynecology. 2018;219(1):B2-B9.

22. Achiron R, Schimmel M, Achiron A, Mashiach S. Fetal mild idiopathic lateral ventriculomegaly: is there a correlation with fetal trisomy? Ultrasound in Obstetrics and Gynecology: The Official Journal of the International Society of Ultrasound in Obstetrics and Gynecology. 1993:3(2):89-92.

23. Glick PL, Harrison MR, Nakayama DK, Edwards MS, Filly RA, Chinn $\mathrm{DH}$, et al. Management of ventriculomegaly in the fetus. The Journal of pediatrics. 1984;105(1):97-105

24. Coleman BG, Langer JE, Horii SC. The diagnostic features of spina bifida: the role of ultrasound. Fetal diagnosis and therapy. 2015;37(3):179-96.

25. Chen Q, Feng Z, Tan Q, Guo J, Tang J, Tan L, et al. Post-hemorrhagic hydrocephalus: recent advances and new therapeutic insights. Journal of the Neurological Sciences. 2017;375:220-30.

26. Bromley B, Frigoletto Jr FD, Benacerraf BR. Mild fetal lateral cerebral ventriculomegaly: clinical course and outcome. American journal of obstetrics and gynecology. 1991;164(3):863-7.

27. Pagani G, Thilaganathan B, Prefumo F. Neurodevelopmental outcome in isolated mild fetal ventriculomegaly: systematic review and meta-analysis. Ultrasound in Obstetrics \& Gynecology. 2014;44(3):254. 


\section{ORIGINAL ARTICLE}

